\title{
Modelo de Drude e a interação da luz com a matéria ${ }^{+*}$
}

Fabiana Botelho Kneubil

IEFC - Instituto Educacional Futuro da Ciência

São Paulo - SP

\section{Resumo}

Neste trabalho, discutimos aspectos das dimensões fenomenológica e teórica do conhecimento físico e o papel dos modelos como ponte unificadora dessas duas facetas do conhecimento. No âmbito do ensino, percebe-se que as teorias ensinadas são pouco relacionadas aos fenomenos do cotidiano. Por isso, enfatizamos a importância do uso de modelos como instrumentos capazes de promover uma ligação entre o mundo abstrato, representado pelas teorias e o mundo palpável, representado pelos fenômenos. Com o objetivo de dar realidade à infinidade de nuances que o conhecimento físico contempla, exploramos um modelo, chamado modelo de Drude, voltado para cursos introdutórios de eletromagnetismo do ensino superior. Este exemplo descreve como é a interação de ondas eletromagnéticas com a matéria. O principal objetivo é explicitar, de maneira mais clara possível, as ligações entre ideias físicas, representadas pela teoria do eletromagnetismo, e o mundo fenomenológico, de modo a possibilitar que professores e estudantes de física tenham uma relação com o conhecimento mais epistemológica e rica.

Palavras-chave: Modelos; Modelo de Drude; Ensino sobre Ciência; Ondas Eletromagnéticas.

\footnotetext{
${ }^{+}$Drude model and the interaction of light with matter

* Recebido: fevereiro de 2020. Aceito: fevereiro de 2021.

${ }^{1}$ E-mails: fkneubil@iefc.org.br
} 


\begin{abstract}
In this work we discuss both phenomenological and theoretical aspects of knowledge in Physics and the role of models as bridges between them. As far as teaching is concerned, one notes that theories taught to students normally bear a very little relation with phenomena in daily life. Therefore we emphasize the importance of using models as tools in order to promote meaningful links between the abstract world represented by theories and the real world, represented by phenomena. With the purpose of stressing the different nuances of physical knowledge, we approached a model, known as Drude model, focused on introductory courses of electromagnetism in higher level. This example describes the interaction between electromagnetic waves and matter. The main goal is to make explicit, as clearly as possible, the links between physical ideas, represented by electromagnetic theory, and the real world, so that physics teachers and students may have an epistemological and rich relationship with Physics.
\end{abstract}

Keywords: Models; Drude Model; Education on Science; Electromagnetic Waves.

\title{
I. Introdução
}

As discussões relativas à inovação curricular vêm crescendo nos últimos anos e existe uma preocupação mundial acerca da introdução de elementos históricos, filosóficos e sociológicos nas aulas de ciências (HFC). Vários autores defendem um ensino não apenas de ciência, mas um ensino sobre a ciência, tal como Matthews (1994), que afirma

\footnotetext{
A história, a filosofia e a sociologia da ciência não têm todas as respostas para essa crise, porém possuem algumas delas: podem humanizar as ciências e aproximá-las dos interesses pessoais, éticos, culturais e políticos da comunidade; podem tornar as aulas de ciências mais desafiadoras e reflexivas, permitindo, deste modo, o desenvolvimento do pensamento crítico; podem contribuir para um entendimento mais integral de matéria científica, isto é, podem contribuir para a superação do "mar de falta de significação" que se diz ter inundado as salas de aula de ciências, onde fórmulas e equações são recitadas sem que muitos cheguem a saber o que significam; podem melhorar a formação do professor auxiliando o desenvolvimento de uma epistemologia da ciência mais rica e mais autêntica, ou seja, de uma maior compreensão da estrutura das ciências bem como o espaço que ocupam no sistema intelectual das coisas (MATTHEWS, 1994, p. 165).
} 
Existem diversas pesquisas atuais em estratégias, metodologias e experiências que possibilitam incluir a natureza e a construção da ciência nos programas (HÖTTECKE; HENKE; RIESS, 2010; RUDGE; HOWE, 2009; VILLANI; DIAS; VALADARES, 2010). Vários países, além do Brasil, passam por reformas nos seus sistemas de ensino nas últimas décadas. Trabalhos têm sido apresentados em conferências europeias ${ }^{2}$, discutindo alternativas metodológicas para uma educação sobre a ciência, inclusive no nível superior (HESTENES; 2006; TSEITLIN; GALILI, 2006; KRANJC, 2006, MATTHEWS, 2007). A abordagem principal é o entendimento da ciência através dos modelos e, outros aspectos do conhecimento científico como a questão da realidade, da fenomenologia, do formalismo matemático, das definições e conceitos, resolução de problemas, teorias e ciência normal.

A dificuldade dos alunos na aprendizagem da física como um todo é outra questão que merece ser apontada. Observa-se que mesmo quando os alunos dos cursos de graduação em física dominam os aspectos formais das teorias têm dificuldades em interpretar fenômenos simples. Parece haver uma grande distância entre a teoria e a realidade e o principal objetivo da física como ciência, que é o de oferecer explicações para os fenômenos naturais, fica enfraquecido diante de uma abordagem com pouco significado e apoiada excessivamente, ou exclusivamente, no formalismo matemático empregado apenas na resolução de listas de exercícios (BREWE; SAWTELLE, 2018). Com uma formação dessa natureza, ficará difícil para o futuro professor partir para novas formas de apresentar os conteúdos de física aos seus alunos. Além disso, observa-se também a escassez de interpretações fenomenológicas dos resultados teóricos e das fórmulas presentes nos livros didáticos.

O ensino de física deveria propiciar um melhor entendimento das dimensões teórica e fenomenológica, bem como distinguir as leis, os conceitos, os fenômenos, as fórmulas, os modelos, as relações entre conceitos como elementos que têm papéis diferentes no corpo do conhecimento científico. Queremos mostrar nesse trabalho que o papel dos modelos na ciência é fundamental e que se usado no ensino pode ser uma ferramenta poderosa que propicia discussões ricas sobre a natureza da ciência (GRECA; MOREIRA, 2001; KOPONEN, 2007). A abordagem explícita de elementos epistemológicos pode aproximar os estudantes à verdadeira prática dos cientistas e de uma atitude relacionada ao 'fazer física' (HANSSON; LEDEN, 2016).

\section{Teoria, Fenômenos e Modelos}

Do ponto de vista da razão, não somos capazes de conhecer a realidade diretamente, e o mundo se mostra para nós através dos fenômenos. A ciência procura uma ordem duradoura que é invisível, e a experiência cotidiana mostra, apenas, coisas efêmeras e transitórias. O entendimento do mundo pode acontecer, e o ingrediente necessário é a

\footnotetext{
2 Em 2006, o GIREP (Groupe International de Recherche sur l'Enseignement de la Physique) ocorrido em Amsterdam, teve como tema principal os modelos, com o título Modeling in Physics and Physics Education.
} 
interpretação das observações por meio de formas simbólicas. Todas as relações intelectuais do ser humano com a "realidade" não são diretas, e sim feitas por construções simbólicas.

Existem várias formas de objetivação da "realidade", o que Cassirer (1979) chama de formas simbólicas ou signos. As formas simbólicas podem ser o mito, a linguagem, a religião, a arte e a ciência. Os signos/símbolos podem ser não apenas linguísticos, mas também artísticos e matemáticos. Com esse enfoque, o aspecto absoluto dos conhecimentos produzidos pela física e pela matemática desaparece e conceitos científicos como átomo, carga, força, massa e campo podem não existir, de fato, na realidade. Construções conceituais são necessárias para interpretar o real, o que torna difícil existir um dado observacional puro, pois a percepção é intrinsecamente carregada da razão, imaginação e de aspectos culturais do observador.

Para Bachelard (1978, p. 32), “... tudo é real, o elétron, o núcleo, o átomo, a molécula, a micela, o mineral, o planeta, o astro, a nebulosa. Em nosso ponto de vista, nem tudo é real da mesma maneira, a substância não tem, em todos os níveis, a mesma coerência: a existência não é uma função monótona; ..."

A construção da ciência é feita com artifícios inteligentes advindos da mente do cientista. Conceitos, ideias e a própria física não são autônomos. Apenas a natureza é autônoma, mas a sua interpretação não o é. Ao invés do cientista dizer que está vendo a natureza quando observa um fenômeno, ele de fato está pensando a natureza de uma determinada maneira. Por isso, a construção da realidade, apenas feita a partir de esquemas mentais, não é fruto de um indivíduo isolado, podendo também ser feita socialmente. Desse modo, não existe uma realidade construída por nós totalmente social/cultural ou totalmente individual/instintiva. Todos estão sujeitos a influências externas, que podem conduzir o pensamento e a forma de ver o mundo.

No caso da física, a interpretação dos fenômenos e a visão de mundo recebem influências tanto vindas de dentro da própria ciência como outras de essência política, religiosa ou ligada a outros aspectos culturais. A relação da física com a realidade é muito complexa. O fato de que ela depende do observador significa que é impossível conhecê-la totalmente, como disse o biólogo inglês Haldane: "o universo é não só muito mais extravagante do que sabemos, mas muito mais extravagante do que podemos saber" (KNELLER, 1980, p. 50).

Em física, a tentativa de se aproximar ao máximo da realidade é feita com o uso do artifício dos modelos, que têm papel de mediadores entre teorias abstratas e fenômenos reais. As teorias, sendo abstrações construídas por nossa razão, não são diretamente aplicáveis às coisas reais. Por outro lado, as medidas experimentais puras não constituem elementos suficientes para o corpo de conhecimento da ciência (PIETROCOLA, 2004, p. 80).

O uso dos modelos é essencial para o pensamento de um físico, pois ele suporta explicações do 'porque' tal fato acontece daquele jeito. Mesmo ele não sendo a realidade, a comunidade científica fica satisfeita com modelos de realidade, na medida em que procura 
relações entre as teorias e os dados empíricos. Eles são como portas de comunicação entre dois mundos: a realidade imediata, sentida através dos fenômenos, e a realidade inatingível, representada pelas teorias físicas.

A dicotomia entre teórico e empírico torna imprescindível o uso de modelos na física. Para Bunge (1974, p. 163), "os modelos teóricos ou ideias representam, de uma maneira mais ou menos simbólica, convencional e indireta, com certa aproximação, alguns traços da constituição e comportamento de sistemas físicos. Todo modelo, assim, é parte de pelo menos uma teoria física". Desse modo, nenhuma teoria física representa exatamente um sistema físico, pois se ela é constituída de conceitos (que Bunge chama de conceitos-chave), e estes conceitos não são empíricos, as teorias não formam as imagens do objeto físico, apenas o representam de maneira articulada e esquemática. Bunge (1974, p.16) ainda define modelo teórico como sendo "um sistema hipotético-dedutivo que concerne a um objeto-modelo, que é, por sua vez, uma representação conceitual esquemática de uma coisa ou de uma situação real ou suposta como tal".

Mostramos, na Fig.1, um esquema que ilustra uma visão científica da natureza, onde existem dois mundos diferentes, o dos fenômenos e o da teoria. O mundo fenomenológico é aquele onde uma realidade imediata e concreta se apresenta e é perceptível pelos sentidos e/ou mensurável pelos instrumentos. O mundo teórico, por outro lado, representa uma realidade não imediata e abstrata, cuja estruturação do pensamento é feita pela matemática, que é altamente lógica, racional e geral. Nesta ilustração, os modelos representam as pontes que unem os fenômenos às teorias, ou seja, se constituem em explicações e imagens da natureza, principalmente, quando se trata de fenômenos microscópicos. Um modelo se utiliza da teoria, forma uma imagem do mundo e permite explicar fenômenos e medidas realizadas na dimensão empírica.

Um exemplo dentro da eletricidade que ilustra a estrutura mostrada na Fig.2 é o efeito Hall, descoberto em 1879 por Edwin Herbert Hall que estudava na Universidade de John Hopkins. Numa época em que o próprio elétron ainda era desconhecido, este efeito revelou-se uma fonte de informação muito importante sobre a condução elétrica. Através de informações teóricas, como o conhecimento dos valores das forças elétrica e magnética, da relação entre a tensão e o campo elétrico, é possível saber características microscópicas de um metal. 


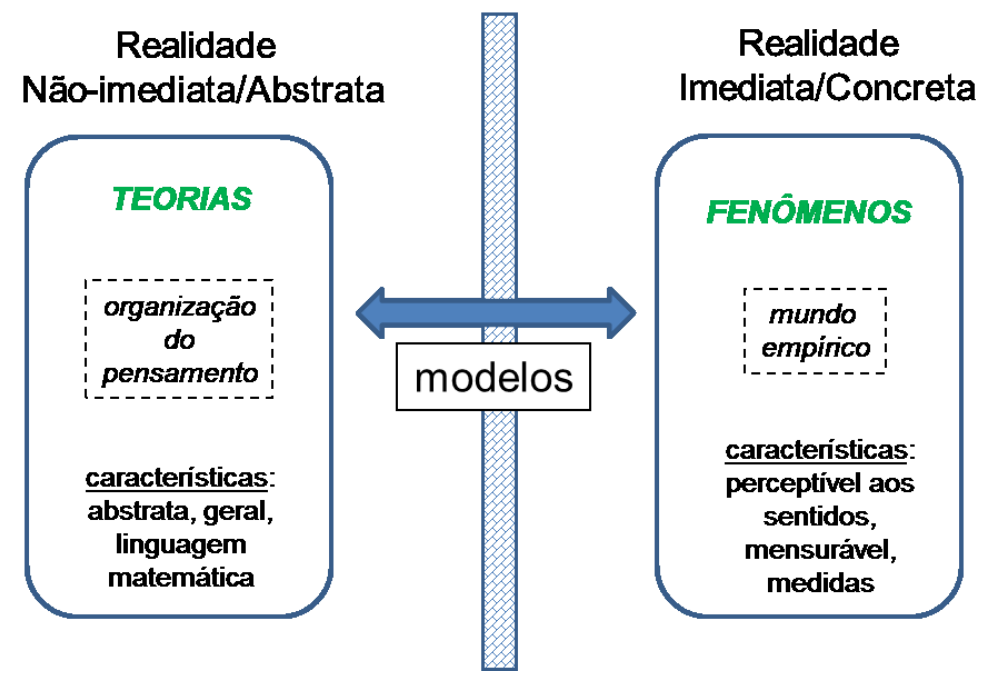

Fig. 1 - Dois mundos: teorias e fenômenos.

A ponte que une a dimensão fenomenológica (medidas experimentais de corrente, diferença de potencial, distância, ...) com a dimensão teórica (expressões das forças e dos campos) é considerada um modelo. Neste caso, usamos o esquema da Fig.1 para o efeito Hall, cujo modelo construído permitiu determinar uma grandeza microscópica, muito importante em eletricidade, que é o número de elétrons livres por volume $\mathrm{N}$, dentro de um metal (KNEUBIL; KARAM, 2017).

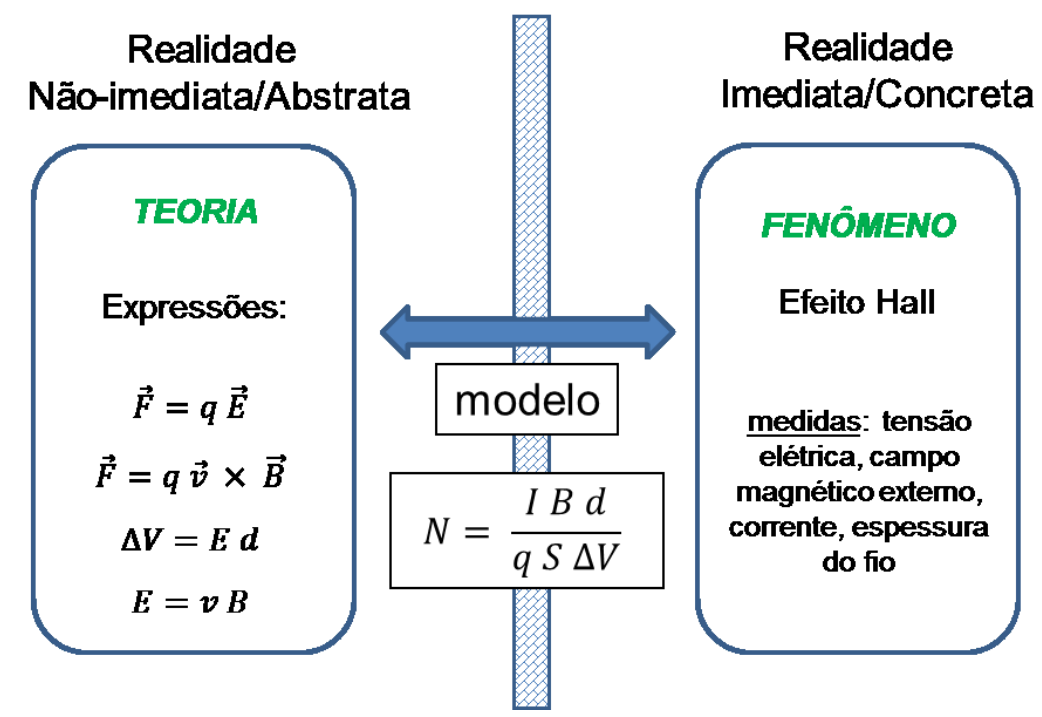

Fig. 2 - Modelo explicativo para o efeito Hall.

\section{O Modelo de Drude e a Interação de Ondas com a Matéria}

Os modelos em física e o uso de estruturas matemáticas para descrevê-los tornaramse cada vez mais complexos. No fim do século 19, Paul Karl Ludwig Drude propôs um 
modelo baseado num raciocínio microscópico ${ }^{3}$ para entender a interação de ondas eletromagnéticas com a matéria. Discutimos, a seguir, o modelo de Drude ${ }^{4}$ aplicado aos metais.

A partir desse modelo, são deduzidas as relações dos campos elétrico e magnético com as grandezas que caracterizam o metal, como a condutividade e a permissividade elétrica do metal.

No interior de um metal, o campo eletromagnético de uma onda é atenuado e parte de sua energia é perdida. Isto se deve à diminuição da amplitude do campo $\vec{E}$, na direção de propagação da onda, por exemplo, ao longo do eixo y. Na Fig. 3(a), temos um esboço do campo elétrico de uma onda no vácuo e, na (b), dentro do metal.

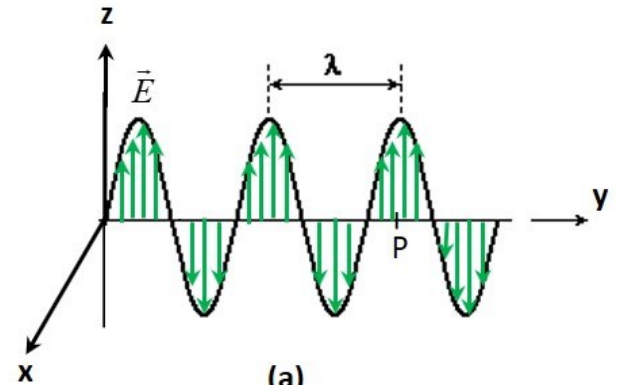

(a)

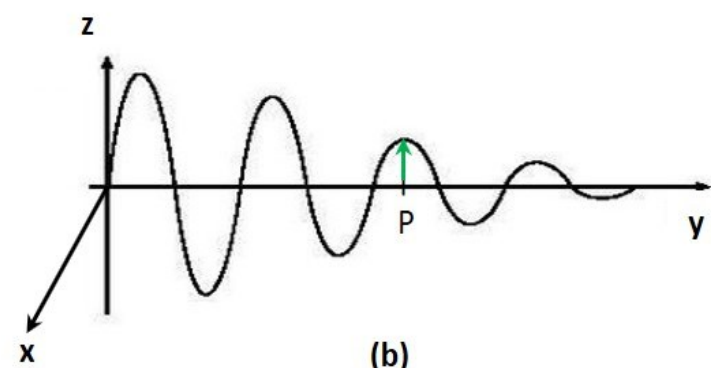

(b)

Fig. 3 - (a) Campo elétrico no vácuo; (b) Campo dentro do metal.

O modelo de Drude nos permite entender como se dá essa atenuação e, por meio de equações para os campos elétrico e magnético, saber como eles se alteram dentro do metal. Consequentemente, também o que acontece com a onda, que é formada por esses campos oscilantes que se propagam. A partir desse conhecimento, podemos explicar os fenômenos de reflexão, refração e transmissão da onda, pois todos eles dependem do resultado da interação desta onda com o meio.

A lei de Ohm em termos do campo elétrico e densidade de corrente é dada por

$$
\vec{J}=\sigma \vec{E},
$$

onde $\sigma$ é a condutividade elétrica do meio. Essa lei só vale para campos elétricos constantes, ou seja, independentes do tempo. Quando existem campos oscilantes no interior do metal, os seus elétrons livres oscilam ${ }^{5}$ e criam outros campos eletromagnéticos também oscilantes ao seu redor. Não temos condições de separar os campos decorrentes do movimento dos elétrons

$3 \mathrm{O}$ modelo de Drude, originalmente, foi desenvolvido para descrever fenômenos relacionados à condução elétrica dentro dos metais. No entanto, ele pôde ser expandido para descrever o comportamento dos elétrons ao serem submetidos a campos advindos das ondas eletromagnéticas.

${ }^{4}$ Esse modelo é abordado no curso de Física 4 do Instituto de Física da USP (BECHARA et al., 2018).

${ }^{5}$ Dentro do metal existem elétrons fortemente ligados ao núcleo e os elétrons livres, responsáveis pela condução. Estamos supondo que apenas os elétrons livres oscilam devido ao campo incidente. 
dos campos que causaram esse movimento. Por isso, no modelo de Drude trabalhamos com a expressão do campo resultante em cada ponto no interior do metal.

Esse campo resultante não é constante ao longo do metal e depende da coordenada y, que é a distância de um ponto qualquer à superfície. Devido ao efeito Joule, é razoável esperarmos que a amplitude desse campo diminua à medida que entramos no metal.

O tratamento matemático do modelo começa com a determinação da relação entre o campo elétrico resultante $\vec{E}$ existente numa região do interior do metal e a densidade de corrente $\vec{\jmath}$ nesse mesmo ponto. No caso de campos estáticos, a densidade de corrente pode ser obtida a partir da eq. (1) e vale

$$
\vec{\jmath}=-e N \vec{v}
$$

onde $\vec{v}$ é a velocidade de arraste dos elétrons, $e$ é a carga elementar e $N$ é o número de portadores de carga por unidade de volume. A eq. (2) tem natureza puramente geométrica e não depende do tipo de força que causa o movimento dos elétrons. No nosso caso, é conveniente usar a forma

$$
\vec{\jmath}=-e N \frac{d \vec{s}}{d t}
$$

onde $\vec{S}$ representa a posição do elétron, que podemos obter usando a segunda lei de Newton. Para tanto, temos que determinar quais forças atuam nos elétrons livres do condutor.

Quando uma onda eletromagnética incide sobre uma superfície metálica, uma parte dela é refletida e outra parte penetra no metal. Supondo que a onda incidente seja plana, monocromática e se propaga na direção y, o campo $\vec{E}_{R}$ resultante num plano que passa por um ponto $\mathrm{P}$, que dista y da superfície externa, dentro do metal, será também oscilante e pode ser escrito como uma função cosseno:

$$
\vec{E}=E_{y} \cos \left(\omega t-\varphi_{y}\right) \hat{k}
$$

onde $E_{y}$ é a amplitude e $\varphi_{y}$ é uma fase genérica, que diferencia o campo incidente do campo no ponto $\mathrm{P}$, como mostrado na Fig.4. Note que $\vec{E}$ está na direção do eixo z, dada pelo versor $\hat{k}$.

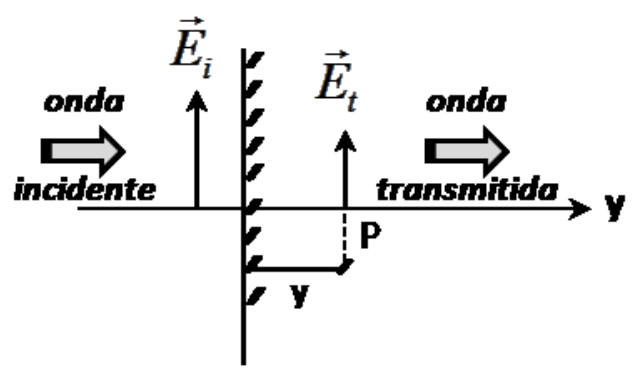

Fig. 4 - Campo elétrico da onda incidente e transmitida. 
Duas forças atuam nos elétrons livres ${ }^{6}$ : uma elétrica e outra de resistência ao movimento, proporcional à velocidade:

$$
b=\frac{N e^{2}}{\sigma},
$$

onde $\sigma$ é a condutividade elétrica do material.

Temos, então, a seguinte equação do movimento do elétron, determinada pela $2^{\mathrm{a}}$ lei de Newton:

$$
m \frac{d^{2} s}{d t^{2}}=-b \frac{d s}{d t}-e E_{y} \cos \left(\omega t-\varphi_{y}\right),
$$

onde $S$ é o seu deslocamento, ao longo do eixo z, em relação à posição de equilíbrio e $m$, a sua massa.

Considerando que a onda já atingiu um regime estacionário, a solução dessa equação é

$$
S=A \cos \left(\omega t-\varphi_{y}+\alpha\right)
$$

Efetuando $d S / d t$ e $d^{2} S / d t^{2}$ e substituindo em (8) ${ }^{7}$, achamos as constantes $A$ e $\alpha$ que representam, respectivamente, o máximo deslocamento do elétron e sua defasagem em relação ao campo elétrico. Elas valem:

$$
A=\frac{e E_{y}}{\omega \sqrt{m^{2} \omega^{2}+b^{2}}}
$$

$\mathrm{e}$

$$
\alpha=\operatorname{arctg}\left(\frac{b}{m \omega}\right) .
$$

Substituindo esse resultado na eq.(9), temos

$$
\vec{S}=\frac{e \vec{E}_{y}}{\omega \sqrt{m^{2} \omega^{2}+b^{2}}} \cos \left(\omega t-\varphi_{y}+\alpha\right) .
$$

Nesse ponto do modelo, podemos fazer uma primeira parada para interpretar os resultados. Partimos de suposições microscópicas para o comportamento do elétron ao ser atingido por uma onda dentro do metal e, usando a lei de Newton, obtivemos uma expressão que descreve esse movimento. Usamos a dimensão teórica do conhecimento científico, representada pelas leis de Newton e de Ohm, para obter uma outra expressão, cujo significado, se interpretado, descreve características que pertencem à dimensão fenomenológica.

A eq.(12), embora complicada, mostra que a frequência $\omega$ da onda está envolvida com dois efeitos, pois ela aparece tanto na amplitude $A$, como na fase da onda. O significado dessa dependência pode ser entendido avaliando os seus respectivos gráficos. Usando os

\footnotetext{
6 Ondas eletromagnéticas são formadas pelos dois campos, elétrico e magnético. No entanto, descrevemos apenas o efeito da força elétrica sob os elétrons, pois a componente magnética é desprezível, como mostraremos mais adiante.
}

${ }^{7}$ Essa resolução pode ser encontrada em KNEUBIL (2006). 
valores calculados das constantes para o cobre e mostrados na tabela 1 (KNEUBIL, 2006), temos os gráficos mostrados na Fig.5.

Tabela 1 - Constantes do cobre.

\begin{tabular}{|l|}
\hline$N=1 \times 10^{29}$ elétrons $/ \mathrm{m}^{3}$ (número de elétrons por volume) \\
\hline$b=4,352 \times 10^{-17} \mathrm{C}^{2} \Omega \mathrm{m}^{-2}$ (coeficiente de atrito) \\
\hline$m=9,11 \times 10^{-31} \mathrm{~kg}$ (massa do elétron) \\
\hline$e=1,6 \times 10^{-19} \mathrm{C}$ (carga elementar) \\
\hline$E_{Y}=80 \mathrm{~V} / \mathrm{m}$ (campo elétrico incidente) ${ }^{8}$ \\
\hline
\end{tabular}

Nesses gráficos, o eixo horizontal representa a frequência da radiação numa escala logarítmica e varre todo o espectro das ondas eletromagnéticas. As radiações conhecidas vão da ordem de $10^{11} \mathrm{~Hz}$, chamadas de radiofrequência, até frequências muito altas, da ordem de $10^{22} \mathrm{~Hz}$, que é o caso dos raios gama.

No gráfico da defasagem é possível perceber a existência de três regiões com características diferentes. Na primeira delas, que corresponde ao intervalo de $0<\omega<10^{10} \mathrm{~Hz}$, a defasagem vale $\pi / 2$, indicando que o campo $\vec{E}$ e a velocidade $d \vec{S} / d t$ estão em fase. No extremo oposto, para frequências maiores do que $10^{18} \mathrm{~Hz}$, a defasagem é praticamente nula e o campo está em fase com o deslocamento do elétron.

No gráfico da amplitude, quando $\omega$ é pequeno, podemos ver que ela se torna muito alta, tendendo a infinito. Isso significa que o elétron se movimenta muito, antes de inverter seu sentido, quando o campo elétrico varia lentamente. Isso é totalmente coerente com o limite $\omega=0$, ou seja, campo estático, que corresponde ao movimento no interior de um fio ligado a uma bateria. Por outro lado, para frequências muito altas, a amplitude tende a zero e o elétron praticamente não se move.

Prosseguindo com o modelo, devemos agora achar a relação entre a densidade de corrente $\vec{\jmath}$ e o campo $\vec{E}$, para, usando as equações de Maxwell, obter as expressões finais dos campos elétrico e magnético dentro da matéria. Derivando a eq.(12) e substituindo em (3), temos que

$$
\vec{\jmath}=\frac{e^{2} N \vec{E}_{y}}{\sqrt{m^{2} \omega^{2}+b^{2}}} \operatorname{sen}\left(\omega t-\varphi_{y}+\alpha\right)
$$

\footnotetext{
${ }^{8}$ Valor estimado do campo elétrico a uma distância de 1 metro de uma lâmpada de 100W.
} 

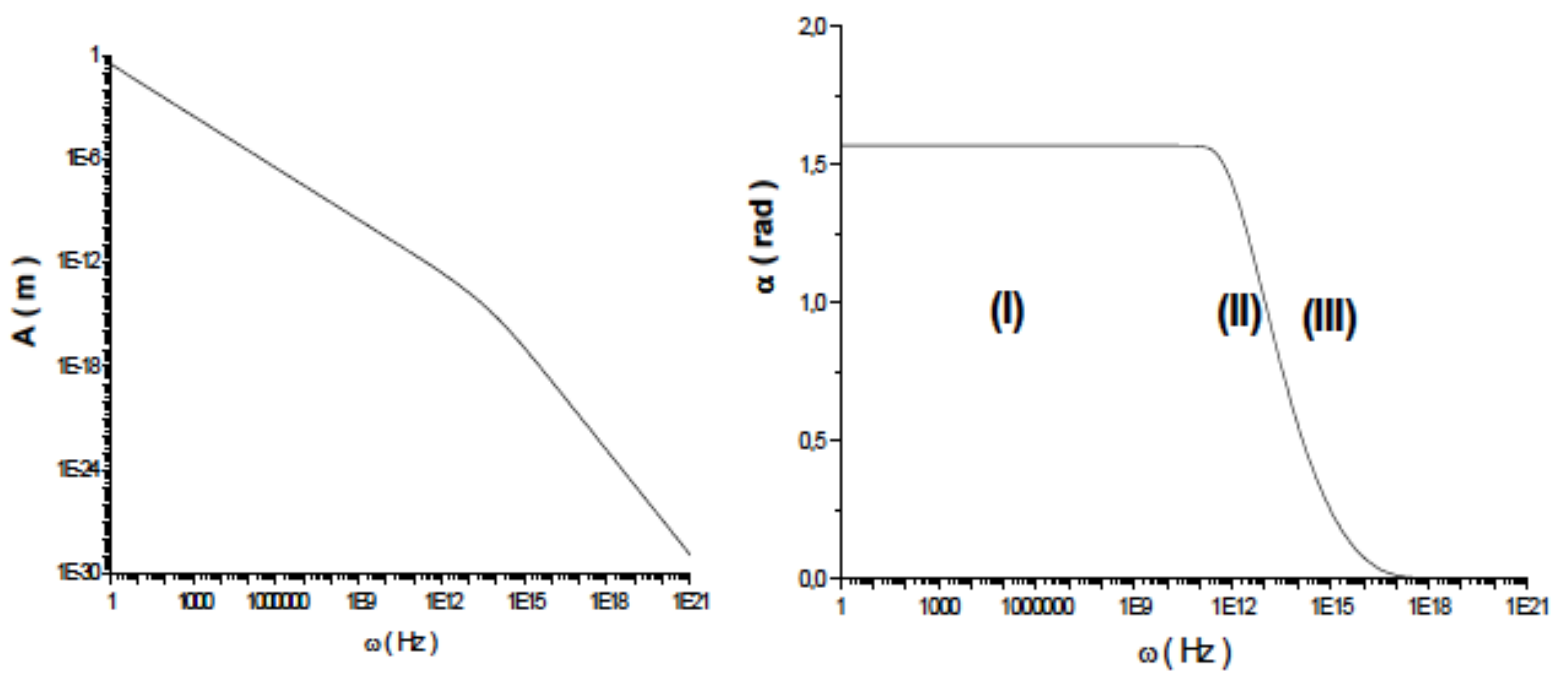

Fig. 5 - Amplitude e defasagem de oscilação do elétron.

Desenvolvendo o seno da soma da equação acima e usando as definições

$$
\operatorname{sen} \alpha=\frac{b}{\sqrt{m^{2} \omega^{2}+b^{2}}} \quad e \quad \cos \alpha=\frac{m \omega}{\sqrt{m^{2} \omega^{2}+b^{2}}},
$$

podemos escrever $\vec{j}$ dividindo em duas partes:

$$
\vec{J}=\vec{J}_{A} \cos \left(\omega t-\varphi_{y}\right)+\vec{J}_{B} \operatorname{sen}\left(\omega t-\varphi_{y}\right),
$$

onde

$$
\vec{J}_{A}=\frac{e^{2} N}{m^{2} \omega^{2}+b^{2}} b \vec{E}_{y} \text { (15) e } \vec{J}_{B}=\frac{e^{2} N m \omega}{m^{2} \omega^{2}+b^{2}} \vec{E}_{y}
$$

O comportamento e o significado dessas duas amplitudes, $\vec{J}_{A}$ e $\vec{J}_{B}$, estão mostrados nos gráficos da Fig. 6.

Nas duas situações limite $\omega \cong 0$ e $\omega \rightarrow \infty$, é mais simples de analisar. No caso, $\omega \cong 0$, pelo gráfico, vemos que $\vec{J}_{B}=0$. Isso corresponde a um campo elétrico constante, como no caso de um fio condutor ligado a uma bateria, pois o segundo termo da eq.(14) desaparece e ela vira a lei de Ohm microscópica $\vec{\jmath}=\sigma \vec{E}$, com $\sigma=\frac{N e^{2}}{b}$, que é a condutividade estática do material. No caso geral, a condutividade é obtida a partir da eq.(15), como uma relação entre $|\vec{\jmath}|$ e $|\vec{E}|$ :

$$
\sigma=\frac{e^{2} N b}{m^{2} \omega^{2}+b^{2}}
$$

sendo dependente da frequência $\omega$ da onda. Entretanto, para valores muito baixos de frequência, o termo $m^{2} \omega^{2}$ é desprezível frente a $b^{2}$ e a expressão (17) se reduz à equação estática. 

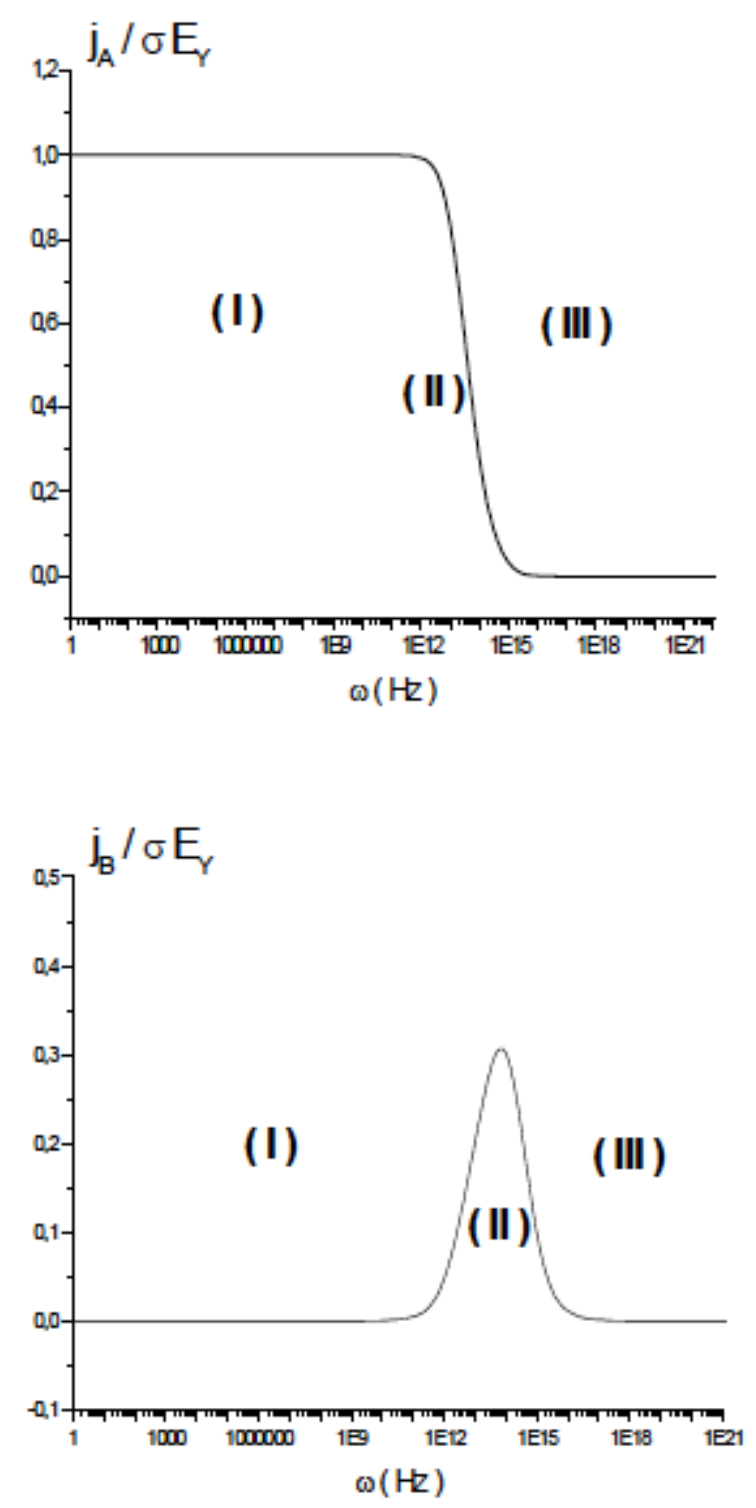

Fig. 6-Amplitude da densidade de corrente.

No regime de frequências baixas, até aproximadamente $10^{12} \mathrm{~Hz}$, a amplitude de $\vec{J}_{A}$ é muito próxima ao valor estático $\sigma \vec{E}_{y}$. A partir daí, conforme $\omega$ aumenta, $\left|\vec{J}_{A}\right|$ diminui, com uma transição que ocorre num intervalo de frequência relativamente pequeno, da ordem de $10^{14} \mathrm{~Hz}$. Do ponto de vista matemático, esse comportamento poderia ter sido visto pela expressão (17), onde os termos $b^{2}$ e $m^{2} \omega^{2}$ concorrem no denominador. Para metais comuns, a ordem de grandeza do coeficiente de atrito $b$ é $10^{-17} \mathrm{C}^{2} \Omega \mathrm{m}^{-2}$ e a massa do elétron é da ordem de $10^{-30} \mathrm{~kg}$. Portanto, para que o produto $m \omega$ seja da ordem de $b$, é preciso que $\omega$ esteja em torno de $10^{13} \mathrm{~Hz}$. Isso determina a faixa de frequência que separa as regiões I e III mostradas nos gráficos, ou seja, corresponde à região II, onde observamos um comportamento bastante complexo da densidade de corrente e sua dependência não apenas com a frequência, mas também com as constantes $b$ e $m$. Assim, na região I, onde $\omega$ é pequeno, o termo $b^{2}$ é 
dominante, na região III é o termo $m^{2} b^{2}$ que prevalece e na região II, a combinação entre $b, m$ e $\omega$ resulta na complexidade da curva.

Para as frequências correspondentes à região I, os elétrons oscilam com amplitude grande e consequentemente, dissipam muito energia, devido aos choques com os íons, e isso corresponde a uma absorção da energia da onda. A consequência disso é o meio material ser opaco, pois a onda não o atravessa para radiações de baixa frequência. Nos gráficos, vemos que existe um intervalo de frequências onde a amplitude e demais grandezas são praticamente constantes e para o qual vale aproximadamente a lei de Ohm.

Para frequências muito altas, como as da região III, as amplitudes de $\vec{J}_{A}$ e $\vec{J}_{B}$ são praticamente nulas, indicando que não existe corrente dentro do material. Nesse caso, a energia da onda não é gasta e ela atravessa o meio facilmente. Assim, o efeito Joule não ocorre, pois os elétrons livres dentro da matéria não se movimentam. Isso mostra que a transparência não é uma característica absoluta de um meio. Portanto, conforme aumentamos a frequência de uma onda, torna-se mais difícil blindar a sua passagem.

Um ponto importante em toda essa análise é o fato de que é a densidade de corrente que rege os acontecimentos dentro da matéria. Para frequências baixas, domina a absorção e para frequências altas, é como se o material não existisse, pois as amplitudes de $\vec{J}_{A}$ e $\vec{J}_{B}$ tendem a zero, dominando, portanto, a transmissão da onda.

No desenvolvimento do modelo de Drude, a densidade de corrente é usada nas equações de Maxwell. Por isso, é conveniente reescrevê-la em função da derivada do campo elétrico. Usando a eq.(4), temos que

$$
\frac{d \vec{E}}{d t}=-\omega \vec{E}_{y} \operatorname{sen}\left(\omega t-\varphi_{y}\right)
$$

e, desenvolvendo a eq.(14), obtemos a relação entre a densidade de corrente, o campo elétrico e a sua derivada temporal:

$$
\vec{\jmath}(\omega)=\frac{N e^{2} b}{m^{2} \omega^{2}+b^{2}} \vec{E}-\frac{N e^{2} m}{m^{2} \omega^{2}+b^{2}} \frac{\partial \vec{E}}{\partial t} .
$$

A expressão acima é parecida com a lei de Ohm para o caso estático, mas possui um termo a mais. Esse termo envolve a variação temporal do campo e é responsável pela propagação da onda dentro do material. Ela é conhecida como lei de Ohm generalizada.

Para achar as expressões dos campos elétrico e magnético dentro do metal, usamos as equações e Maxwell com a densidade de corrente $\vec{\jmath}$ obtida.

$$
\begin{gathered}
\vec{\nabla} \cdot \vec{E}=\frac{\rho}{\varepsilon_{0}}, \\
\vec{\nabla} \times \vec{E}=-\frac{\partial \vec{B}}{\partial t}, \\
\vec{\nabla} \times \vec{B}=\mu_{0} \vec{J}+\varepsilon_{0} \mu_{0} \frac{\partial \vec{E}}{\partial t}, \\
\vec{\nabla} \cdot \vec{B}=0
\end{gathered}
$$


Mesmo estando dentro de um meio material, a densidade de carga, em média, é nula, pois numa determinada região, o número de elétrons é igual ao de prótons. Por isso, na eq.(20), $\rho=0$. Substituindo $\vec{\jmath}$ na eq.(22) e juntando os termos semelhantes em $\vec{E}$ e $\frac{\partial \vec{E}}{\partial t}$, podemos reescrever as quatro equações:

$$
\begin{gathered}
\vec{\nabla} \cdot \vec{E}=0, \\
\vec{\nabla} \times \vec{E}=-\frac{\partial \vec{B}}{\partial t}, \\
\vec{\nabla} \cdot \vec{B}=0, \\
\vec{\nabla} \times \vec{B}=\mu_{0} \sigma(\omega) \vec{E}+\mu_{0} \varepsilon(\omega) \frac{\partial \vec{E}}{\partial t},
\end{gathered}
$$

onde definimos

$$
\sigma(\omega)=\frac{e^{2} N b}{m^{2} \omega^{2}+b^{2}} \quad \text { (28) e } \varepsilon(\omega)=\varepsilon_{0}-\frac{m e^{2} N}{m^{2} \omega^{2}+b^{2}} .
$$

As grandezas $\sigma(\omega)$ e $\varepsilon(\omega)$ são, respectivamente, a condutividade e permissividade elétrico do meio e dependem da frequência $\omega$ da onda incidente. Para desacoplar os campos $\vec{E}$ e $\vec{B}$ das equações de Maxwell, calculamos o rotacional das expressões (25) e (27) e chegamos às equações de onda do campo elétrico e magnético no interior do metal. Elas são dadas por

$$
\begin{aligned}
& \frac{\partial^{2} \vec{E}}{\partial x^{2}}+\frac{\partial^{2} \vec{E}}{\partial y^{2}}+\frac{\partial^{2} \vec{E}}{\partial z^{2}}-\mu_{0} \varepsilon(\omega) \frac{\partial^{2} \vec{E}}{\partial t^{2}}=\mu_{0} \sigma(\omega) \frac{\partial \vec{E}}{\partial t} \\
& \frac{\partial^{2} \vec{B}}{\partial x^{2}}+\frac{\partial^{2} \vec{B}}{\partial y^{2}}+\frac{\partial^{2} \vec{B}}{\partial z^{2}}-\mu_{0} \varepsilon(\omega) \frac{\partial^{2} \vec{B}}{\partial t^{2}}=\mu_{0} \sigma(\omega) \frac{\partial \vec{B}}{\partial t} .
\end{aligned}
$$

Essas equações têm duas diferenças importantes em relação às equações de onda no vácuo. Os termos $\partial \vec{E} / \partial \mathrm{t}$ e $\partial \vec{B} / \partial \mathrm{t}$ do lado direito nas equações, referentes à variação temporal dos campos em primeira ordem, estão relacionado à absorção da onda dentro do meio. A segunda diferença é que a permissividade $\varepsilon(\omega)$ passa a ser variável e depende da frequência $\omega$ da onda. Essa grandeza está relacionada com a existência de um índice de refração para o meio, que também depende da frequência da onda.

No interior do metal, as quatro equações acima se reduzem as do vácuo, pois numa região em que não existem elétrons livres, o valor de $\mathrm{N}$ é nulo, e as equações (28) e (29) resultam em

$$
\lim _{N \rightarrow 0} \sigma(\omega)=0
$$

$\mathrm{e}$

$$
\lim _{N \rightarrow 0} \varepsilon(\omega)=\varepsilon_{0}
$$

As equações de onda dos campos $\vec{E}$ e $\vec{B}$ descrevem, em princípio, ondas tridimensionais, que podem ser bastante complicadas. Consideramos um caso mais simples, no qual as ondas são planas e se propagam ao longo do eixo y, o que nos permite escrever 
$\vec{E}(x, y, z, t)=\vec{E}(y, t)$. Por isso, as eqs. (30) e (31) ficam mais simples e podem ser escritas como

$$
\begin{aligned}
& \frac{\partial^{2} \vec{E}}{\partial y^{2}}-\mu_{0} \varepsilon(\omega) \frac{\partial^{2} \vec{E}}{\partial t^{2}}=\mu_{0} \sigma(\omega) \frac{\partial \vec{E}}{\partial t} \\
& \frac{\partial^{2} \vec{B}}{\partial y^{2}}-\mu_{0} \varepsilon(\omega) \frac{\partial^{2} \vec{B}}{\partial t^{2}}=\mu_{0} \sigma(\omega) \frac{\partial \vec{B}}{\partial t} .
\end{aligned}
$$

Primeiro, resolvemos o caso do campo elétrico. O fato de que, dentro do metal, o campo $\vec{E}$ ao mesmo tempo oscila e sofre uma atenuação, sugere que a solução da equação (34) seja do tipo

$$
\vec{E}=\vec{E}_{0} \mathrm{e}^{-\beta y} \cos (k y-\omega t+\theta),
$$

onde $\beta$ e $\mathrm{k}$ são constantes a ser determinadas. Para isso, derivamos a equação acima em relação às variáveis t e y, e substituímos em (34). Além disso, para obter a expressão do campo magnético, utilizamos a lei de Faraday (eq. 25), calculando separadamente $\vec{\nabla} \times \vec{E}$ e igualando a $-\partial \vec{B} / \partial$ t. Apresentamos, a seguir, diretamente os resultados obtidos desses cálculos.

$$
\begin{gathered}
\vec{E}=\vec{E}_{0} \mathrm{e}^{-\beta \mathrm{y}} \cos (k y-\omega t+\theta) \\
\beta=\frac{\mu_{0} \omega \sigma(\omega)}{2 k} \\
k=\mp \sqrt{\mu_{0}} \omega \sqrt{\frac{\varepsilon(\omega)}{2}+\frac{1}{2} \sqrt{\varepsilon^{2}(\omega)+\frac{\sigma^{2}(\omega)}{\omega^{2}}}} \\
\sigma(\omega)=\frac{e^{2} N b}{m^{2} \omega^{2}+b^{2}} \\
\vec{B}_{\vec{B}}=\overrightarrow{\mathrm{B}}_{0} \mathrm{e}^{-\beta \mathrm{y}} \cos \left(k y-\frac{m e^{2} N}{m^{2} \omega^{2}+b^{2}}\right. \\
\vec{B}_{0}=\frac{\sqrt{\mathrm{k}^{2}+\beta^{2}}}{\omega} \vec{\jmath} \times \vec{E}_{0} \\
\operatorname{tg} \eta=\frac{\beta}{k}
\end{gathered}
$$

\section{Discussão dos resultados}

Após todos os cálculos matemáticos, o resultado obtido com esse modelo deve explicar aspectos fenomenológicos da interação de uma onda com a matéria. Partimos de uma dimensão teórico-abstrata, usando as leis de Ohm, de Newton, equações de Maxwell e chegamos nas expressões que podem explicar 'coisas' relativas à dimensão fenomenológica, que é menos abstrata, mais concreta e palpável que a dimensão teórica. 
Na física, os modelos têm esse papel. No caso do efeito Hall, citado anteriormente, a matemática envolvida é simples e ele possui uma face com a dimensão fenomenológica maior. O modelo de Drude é bem mais elaborado e, claramente, a manipulação da teoria e a matemática envolvida são mais complexas. Por isso, a dificuldade na interpretação e na adequação à dimensão fenomenológica é muito grande. Em geral, a explicação mais profunda dos fenômenos advindos da interação de ondas com a matéria não é feita nem em aulas dos cursos de graduação, nem aparecem nos livros didáticos. Queremos agora mostrar como é essa interpretação e aplicar esses resultados.

Nesse modelo, as cinco grandezas mais importantes que descrevem o comportamento da onda dentro do metal e o movimento dos elétrons livres são: a posição do elétron $\vec{S}$, sua velocidade $\vec{v}$, a densidade de corrente $\vec{\jmath}$, o campo elétrico $\vec{E}$ e o campo magnético $\vec{B}$.

Um aspecto relevante das expressões dos campos obtidos com o modelo é que elas representam os campos resultantes num ponto qualquer dentro do metal. Não há como separar o campo da onda incidente do campo gerado pela oscilação do elétron e, portanto, aquelas expressões dizem respeito à soma desses dois campos naquele ponto. Fazendo uma retrospectiva, a construção do modelo foi iniciada com a ideia de que o...

... campo elétrico oscilante $\vec{E}$ age sobre o elétron, fazendo com que ele também oscile. Consequentemente, sua posição, dada por $\vec{S}$, que varia com o tempo, causa uma corrente elétrica $\vec{\jmath}$, proporcional a $\partial \vec{S} / \partial \mathrm{t}$. Essa densidade de corrente elétrica $\vec{\jmath}$, juntamente com a variação do campo elétrico $\partial \vec{E} / \partial$ t, gera um campo magnético $\vec{B}$, juntamente com a variação do campo elétrico $\partial \vec{E} / \partial$ t, gera um campo magnético $\vec{B}$, também oscilante. E este, por sua vez, gera o mesmo...

... campo elétrico oscilante $\vec{E}$ age sobre o elétron, fazendo com que ele também oscile. Consequentemente, sua posição, dada por $\vec{S}$, que varia com o tempo, causa uma corrente elétrica $\vec{\jmath}$, proporcional a $\partial \vec{S} / \partial \mathrm{t}$. Essa densidade de corrente elétrica $\vec{\jmath}$, juntamente com a variação do campo elétrico $\partial \vec{E} / \partial \mathrm{t}$, gera um campo magnético $\vec{B}$, também oscilante. E este, por sua vez, gera o mesmo...

Através dessa brincadeira de repetir propositalmente os dois parágrafos acima, queremos chamar a atenção para o fato de que o modelo de Drude envolve uma forma complexa de auto-consistência. Os dois campos $\vec{E}$, o incidente e o advindo da oscilação do elétron, somados, são sempre iguais ao campo resultante, o que vale também para o campo $\vec{B}$.

\section{(a) Defasagens}

As expressões (37) e (42) representam os campos elétrico e magnético dentro do metal. $\mathrm{O}$ termo $\mathrm{e}^{-\beta \mathrm{y}}$ é responsável pelo amortecimento da onda, à medida que ela se propaga. De modo geral, a constante $\beta$ depende do material e da frequência da onda.

$\mathrm{O}$ termo $\cos (k y-\omega t+\theta)$, por outro lado, está relacionado à propagação do campo e descreve a onda em qualquer coordenada y dentro do metal. 
É importante percebermos que existem três fases envolvidas nas expressões dos campos. Ao atravessar a interface, o campo sofre uma defasagem $\theta$. A partir da superfície do lado de dentro até um ponto $\mathrm{P}$ qualquer, há uma defasagem devido à mudança de coordenada y, que vale ky. A Fig. 7 mostra o significado dessas defasagens. Temos, ainda, que $\eta$ é a defasagem entre $\vec{E}$ e $\vec{B}$, obtida pela eq. (44). Ela implica matematicamente que, se a amplitude de um deles é máxima num ponto y qualquer, a do outro não será nesse mesmo ponto.

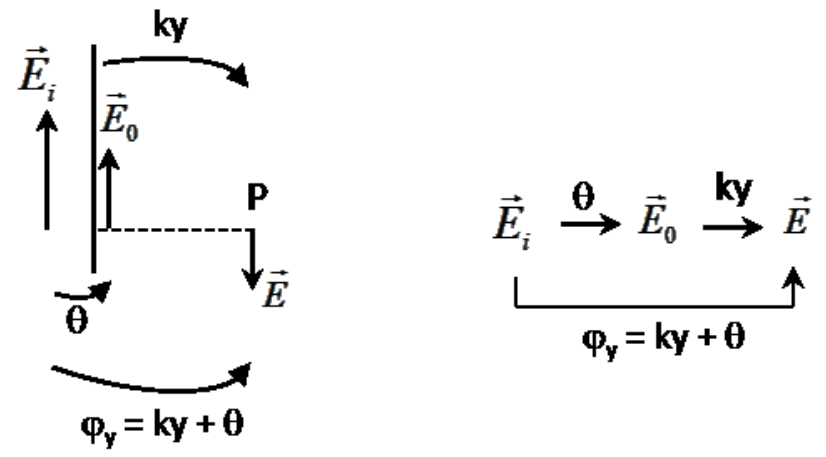

Fig. 7 -Defasagens.

\section{(b) Permeabilidade magnética}

Observando as equações de onda dos dois campos $\vec{E}$ e $\vec{B}$ dentro do material, vemos que na eq.(41), a permissividade elétrica $\varepsilon$ é diferente da do vácuo e depende da frequência $\omega$. Por quê, dentro do metal, a permeabilidade magnética $\mu_{0}$ também não é variável e dependente da frequência?

Quando analisamos as forças que atuam nos elétrons livres, para obter a equação do movimento por meio da lei de Newton, não consideramos a força magnética. A força de Lorentz é dada por

$$
\vec{F}=\mathrm{q}(\overrightarrow{\mathrm{E}}+\vec{v} \times \vec{B}) .
$$

Para o termo magnético ser considerado, ele precisa ter a mesma ordem de grandeza que o elétrico. Para um caso concreto, usando os valores das constantes do cobre e uma onda de frequência $\omega=10^{9} \mathrm{~Hz}$, os parâmetros $\beta$ e $\mathrm{k}$ do modelo são: $\mathrm{k}=1,922 \times 10^{5} \mathrm{~m}^{-1}, \beta=$ $1,922 \times 10^{5} \mathrm{~m}^{-1}$. Adotando $|\overrightarrow{\mathrm{E}}|=\mathrm{E}_{0}=80 \mathrm{~V} / \mathrm{m}$, os módulos da velocidade do elétron (derivada da eq. 12) e do campo magnético da eq.(43) são:

$$
\begin{aligned}
& |\vec{v}|=\frac{e E_{0}}{\sqrt{m^{2} \omega^{2}+b^{2}}}=3,7 \cdot 10^{-2} \mathrm{~m} / \mathrm{s} \\
& |\vec{B}|=E_{0} \frac{\sqrt{\mathrm{k}^{2}+\beta^{2}}}{\omega}=2,7 \cdot 10^{-3} \mathrm{~T},
\end{aligned}
$$

e, portanto, $|\vec{v} \times \vec{B}| \cong 10^{-4} \mathrm{~V} / \mathrm{m}$. Isso mostra que a força elétrica é cerca de cem mil vezes maior que a magnética. Como consequência, a densidade de corrente $\vec{\jmath}$ não depende de $\vec{B}$ e a 
permeabilidade magnética do metal praticamente não se altera e pode ser considerada igual à do vácuo, cujo valor é $\mu_{0}=4 \pi \cdot 10^{-7} \mathrm{H} / \mathrm{m}$.

\section{(c) Espessura de penetração da onda}

A função exponencial $\mathrm{e}^{-\beta y}$ desempenha um papel muito importante nas expressões dos campos, pois representa o amortecimento da onda dentro da matéria. É comparando as constantes $\beta$ e k que podemos saber se domina a absorção ou a propagação, isto é, se $\beta>\mathrm{k}$, o amortecimento predomina, se $\beta<\mathrm{k}$, a onda se propaga. Essa função determina a fração da energia do campo que é absorvida. Essa absorção é caracterizada por uma distância ao longo do eixo y, chamada espessura de penetração $\delta$, que corresponde ao ponto onde o campo elétrico foi reduzido a $37 \%$ do seu valor inicial. Para que a exponencial valha $1 / \mathrm{e}=0,37$, essa distância deve ser o inverso da constante $\beta$, de modo que $\mathrm{e}^{-\beta \delta}=\mathrm{e}^{-1}$. Assim, $\delta=1 / \beta$. Usando os valores para o caso do cobre, construímos, na Fig.8, o gráfico de $\delta$ em função de $\omega$.

Podemos notar que a curva tem uma mudança de comportamento na região do seu mínimo, que ocorre em frequências da ordem de $10^{16} \mathrm{~Hz}$. Para entender o significado físico dessa mudança, temos que imaginar o que acontece com o elétron dentro do material e interpretar fisicamente as expressões resultantes do modelo. Vamos considerar dois regimes, de baixas e altas frequências.

\section{- Frequências Baixas}

Nesse regime, a espessura de penetração diminui uniformemente. Analisando a eq. (19) para baixas frequências, no denominador dos dois termos, o produto $m^{2} \omega^{2}$ é desprezível frente a $b^{2}$. Do mesmo modo, no numerador do segundo termo, o produto da massa do elétron por $\partial \vec{E} / \partial \mathrm{t}$ é proporcional a $m \omega$ e também pode ser desprezado. Com isso, as eq. (40) e (41) se reduzem a $\sigma=\frac{e^{2} N}{b}$ e $\varepsilon=\varepsilon_{0} \mathrm{e}$, portanto, descrevem o caso estático. Esses termos que são desprezados nos permitem entender que para baixas frequências a massa do elétron também é desprezível. Conforme a frequência da onda aumenta, o termo $m^{2} \omega^{2}$ passa a ser importante e a curva muda de qualidade.

\section{- Frequências Altas}

Para frequências maiores que $10^{14} \mathrm{~Hz}$, a massa do elétron começa a ser relevante, o que indica que os efeitos da sua inércia aparecem na oscilação. Todas as grandezas tornam-se bastante complicadas, pois nenhum termo mais pode ser desprezado.

Do ponto de vista físico-microscópico, o fato da massa do elétron explicar o aumento da espessura de penetração significa que a sua inércia "atrapalha" a oscilação. Sendo a frequência do campo elétrico alta, ele alterna suas fases muito rapidamente, e não "dá tempo" para o elétron responder à solicitação do campo. Assim, com o elétron se movendo pouco, a 


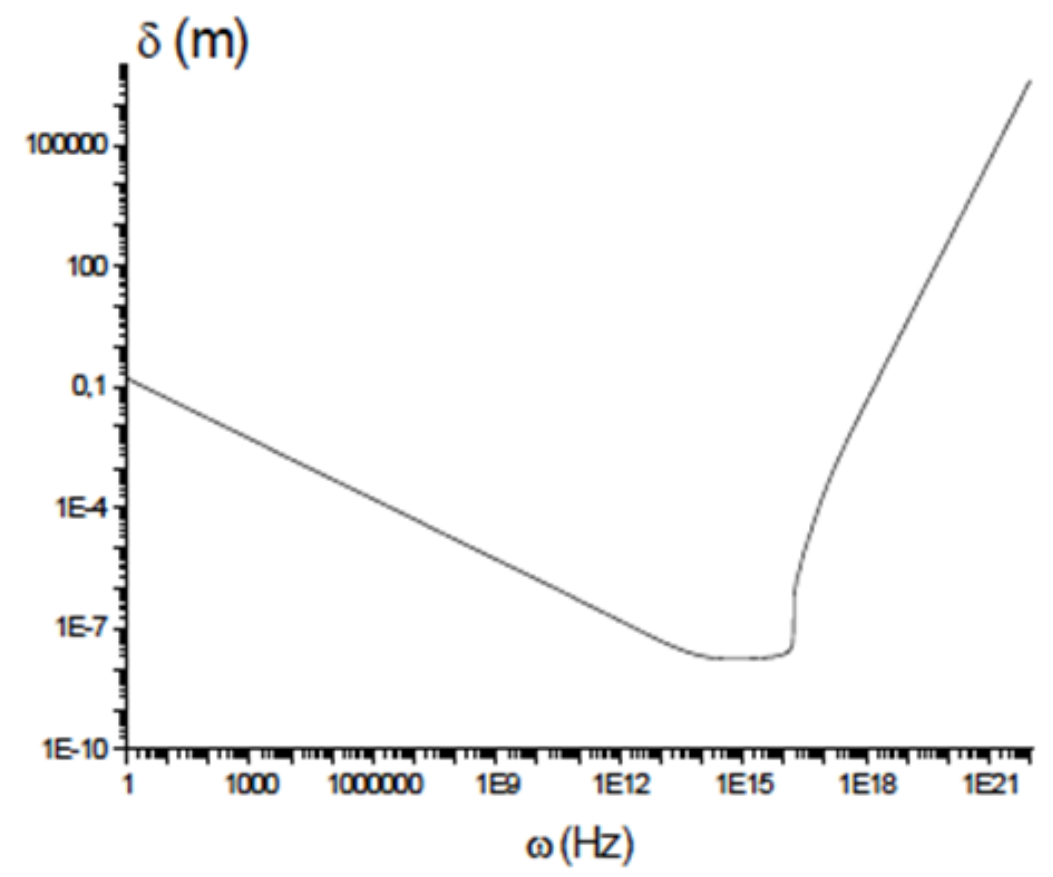

Fig. 8 - Espessura de penetração.

energia da onda não é dissipada e ocorre a transparência do objeto para altas frequências. Intuitivamente, isso explica porque quanto maior a frequência da onda, maior penetração ela terá dentro do material.

\section{- Frequência de Plasma}

A região $10^{13} \mathrm{~Hz}<\omega<10^{17} \mathrm{~Hz}$, que marca a transição entre os regimes de baixa e alta frequência, contém a chamada frequência de plasma. Conceitualmente, essa frequência é muito importante, pois divide as regiões onde dominam o amortecimento e a propagação da onda 9 .

Dissemos anteriormente que o domínio do amortecimento ou da propagação dependem das funções $\beta(\omega)$ e $\mathrm{k}(\omega)$. Se $\mathrm{k}>\beta$, a propagação domina e, matematicamente, isso corresponde a valores positivos de $\varepsilon(\omega)$. Por outro lado, se $\mathrm{k}<\beta$, corresponde a uma atenuação da onda, onde $\varepsilon(\omega)$ deve ser negativo. A relação entre k e $\varepsilon$, descrita pela eq.(39), é bastante complexa, do ponto de vista matemático, o que evidencia a complexidade do modelo de Drude. No entanto, a interpretação física desta dimensão matemática-teórica pode ser feita com base no cálculo de todas as grandezas em função da frequência, apresentado em uma tabela e seus correspondentes gráficos no apêndice 1 .

A expressão teórica que determina a frequência de plasma é obtida impondo a condição $\varepsilon\left(\omega_{\mathrm{p}}\right)=0$. Assim,

\footnotetext{
${ }^{9}$ Uma discussão aprofundada sobre o comportamento matemático da espessura de penetração neste intervalo de frequências pode ser encontrada em Kneubil (2006).
} 


$$
\varepsilon\left(\omega_{p}\right)=\varepsilon_{0}\left(1-\frac{e^{2} \frac{N}{m \varepsilon_{0}}}{\omega_{p}^{2}+\frac{b^{2}}{m^{2}}}\right)=0
$$

o que corresponde a

$$
\omega_{p}^{2}=\frac{e^{2} N}{m \varepsilon_{0}}-\frac{b^{2}}{m^{2}} .
$$

Para a maioria dos metais, o segundo termo da equação acima é muito menor que o primeiro. Portanto, podemos desprezá-lo e a frequência de plasma pode ser escrita como

$$
\omega_{p}=\sqrt{\frac{e^{2} N}{m \varepsilon_{0}}} .
$$

Para o cobre, usando novamente os valores das suas constantes, obtemos que $\omega_{\mathrm{p}}=1,79$ x $10^{16} \mathrm{~Hz}$. Na tabela 2, mostramos alguns valores reais de frequência, espessura de penetração e comprimento de onda.

Tabela 2 - Espessura de penetração e comprimento de onda para algumas frequências.

\begin{tabular}{|c|c|c|c|c|}
\hline radiação & $\omega(\mathrm{Hz})$ & $\beta / \mathrm{k}$ & $\delta(\mathrm{m})$ & $\lambda(\mathrm{m})$ \\
\hline infravermelho & $10^{13}$ & 1,23 & $4,8 \times 10^{-8}$ & $3,71 \times 10^{-7}$ \\
\hline ultravioleta & $10^{16}$ & 1 & $4,6 \times 10^{-8}$ & $2,89 \times 10^{-6}$ \\
\hline raio X & $10^{19}$ & $10^{-11}$ & 3,95 & $1,88 \times 10^{-10}$ \\
\hline raios gama & $10^{22}$ & $10^{-20}$ & $3,95 \times 10^{6}$ & $1,88 \times 10^{-13}$ \\
\hline
\end{tabular}

Podemos observar que para as duas primeiras frequências, que são baixas, $\delta$ tem valores muito pequenos. Para $\omega=10^{19} \mathrm{~Hz}$, a espessura de penetração vale, aproximadamente, 4 metros e, portanto, nesse ponto, o valor da amplitude do campo elétrico é $37 \%$ do inicial. Para $\omega=10^{22} \mathrm{~Hz}$, o valor de $\delta$ aumenta muito, chegando a 4 mil quilômetros (quase metade do raio da Terra!), o que significa que radiações de alta frequência são muito difíceis de serem blindadas.

\section{Alguns fenômenos}

Após toda essa discussão sobre os aspectos das ondas eletromagnéticas e sua interação com a matéria gostaríamos de mostrar como os resultados produzidos podem contribuir para explicar alguns fenômenos visíveis. Um ponto importante muito geral, ilustrado pelo modelo de Drude é que as grandezas físicas que descrevem o comportamento 
eletromagnético e, consequentemente, óptico de um sistema, dependem da frequência da onda incidente.

Vimos que as expressões resultantes do modelo de Drude, além de complicadas, são dependentes umas das outras. A relação entre os campos $\vec{E}$ e $\vec{B}$ e as funções $k, \beta, \sigma$ e $\varepsilon$ são totalmente vinculadas entre si e dependem das características do material, tais como número de elétrons por unidade de volume $N$, massa do elétron $m$ e coeficiente de atrito $b$ que o meio oferece ao movimento dos elétrons.

Apresentamos, a seguir, alguns fenômenos que estão relacionados com esses aspectos da onda que acabamos de mencionar.

As informações acerca da propagação ou amortecimento de uma onda no interior de um metal estão contidas na expressão do campo elétrico $\vec{E}=\vec{E}_{0} \mathrm{e}^{-\beta y} \cos (k y-\omega t+\theta)$ e nas funções $\mathrm{k}$ e $\beta$. A propagação do campo se dá ao longo do eixo y e o valor de y na expressão representa a distância do ponto considerado à interface. Para um metal ser transparente a uma determinada radiação, a espessura de penetração $\delta$ deve ser maior que a espessura do próprio metal. No caso da maioria dos metais, a espessura de penetração é muito pequena, podendo chegar a $0,6 \mathrm{~nm}$, dependendo de $\omega$. Isso acontece para frequências menores do que aproximadamente $10^{18} \mathrm{~Hz}$ e, por isso, os metais são opacos na faixa da luz visível. Se a luz não os atravessa, ela será refletida e o brilho metálico é resultado dessa reflexão. A maior parte da energia da onda incidente é carregada pela onda refletida e, como resultado, metais como o alumínio, o estanho e o aço têm uma aparência cinzenta-brilhante. Eles refletem a luz incidente, independentemente da faixa de frequência e são, portanto, incolores.

A reflexão que promove o brilho em metais e é responsável pela formação de imagens é chamada reflexão especular. Ela só ocorre em superfícies lisas e polidas, de forma que os raios de luz que chegam paralelos entre si, também saiam da mesma forma. Para a prata, que é o material normalmente utilizado em espelhos, uma fina película, apenas maior que a espessura de penetração, é suficiente para impedir que a luz o atravesse e tornar a superfície refletora, capaz de formar imagens de objetos. Entretanto, diminuindo a película, de forma que ela fique comparável com a espessura de penetração, a reflexão da luz deixa de prevalecer e parte dessa luz também a atravessa. É o que acontece com os óculos "espelhados", eles escurecem (diminuem a intensidade da luz transmitida) e refletem ao mesmo tempo. Para que isso seja possível, a espessura da camada metálica que constitui os óculos deve ser da ordem de $10^{-8} \mathrm{~m}$, que corresponde a aproximadamente 50 átomos ${ }^{10}$.

Se uma superfície de prata não for lisa, mas sim rugosa e irregular, a luz incidente sofrerá uma reflexão difusa. Nessa reflexão, os raios que chegam em feixes não saem paralelos e, por isso não vale mais a lei da reflexão de maneira geral ${ }^{11}$. Essa superfície,

\footnotetext{
$10 \mathrm{O}$ cálculo de quantos átomos existem em 1 metro pode ser encontrado em Kneubil (2006).

11 É importante lembrar que cada raio individual obedece a lei da reflexão, mas como são muitos ângulos diferentes formados pela superfície irregular, os raios refletidos terão direções diferentes.
} 
diferentemente do espelho, não formará imagens e não será brilhante. É a reflexão difusa ou a especular que diferencia um metal cinza opaco ${ }^{12}$ de outro cinza metálico e brilhante. Assim, é a forma da interface, lisa ou rugosa, que define o seu brilho, e não o material ${ }^{13}$.

Um aspecto interessante desta discussão é que ela mostra que características de materiais tais como a sua transparência (que corresponde a uma maior transmissão da luz incidente) e a sua opacidade (que corresponde a uma maior reflexão ou absorção da luz incidente) resultam de um jogo entre a espessura de penetração $\delta$ e a espessura do material. Se a espessura do metal for muito grande, a luz reflete, se ela for fina, a luz atravessa.

Encontramos, também, alguns metais que possuem cores, como o ouro, amarelado e o cobre, avermelhado. Para o cobre ${ }^{14}$, na faixa de frequência do visível, o valor de $k$ aumenta com o aumento da frequência $\omega$ e, consequentemente, há mais transmissão da onda. Isso significa que quando uma luz branca incide num pedaço de cobre ou ouro, as cores de maior frequência serão mais transmitidas. Assim, o cobre e o ouro refletem as cores de menor frequência, como o amarelo e o vermelho. Na Fig.9, vemos o índice de reflectância em função do comprimento de onda da radiação incidente, para o alumínio, a prata, o cobre e o ouro.

A faixa escura representa o intervalo de frequências da luz visível. É interessante observar que, para o cobre e para o ouro, a reflectância é maior para frequências menores (amarelo, laranja e vermelho). Já para o alumínio e a prata, a reflectância é alta e praticamente igual para todas as frequências da faixa visível, o que explica a ausência de cor desses metais. De modo geral, para frequências abaixo do visível (infravermelho), os quatro metais são opacos, pois o índice de reflectância é grande. Para frequências maiores que o visível (ultravioleta), apenas o alumínio é opaco e, o ouro, o cobre e a prata, por refletirem pouco dessa radiação, são transparentes a ela.

É importante dizer que, embora o modelo de Drude propicie um entendimento de características físicas dos metais, ele possui algumas limitações em relação a algumas outras propriedades, como as cores, que só podem ser entendidas com modelos que envolvam a natureza quântica da luz (SCARINCI e MARINELI, 2014).

\section{Considerações finais}

Embora haja muita pesquisa sobre a inserção de elementos de epistemologia no ensino de física, especificamente o uso de modelos, observa-se que ainda existe uma grande lacuna entre os resultados dessas pesquisas e a prática efetiva em sala de aula. Durante quase todo o

\footnotetext{
$12 \mathrm{Na}$ prática, é comum que metais também pareçam opacos devido à existência de óxidos nas suas superfícies.

13 A natureza do material define quais frequências serão refletidas e quais serão absorvidas, é o que chamamos óptica física. A forma do material define se valerá a lei da reflexão $\left(\theta_{\mathrm{I}}=\theta_{\mathrm{R}}\right)$, é o que chamamos de óptica geométrica.

${ }^{14}$ Uma tabela completa com os valores para o cobre pode ser encontrada em Kneubil (2006).
} 


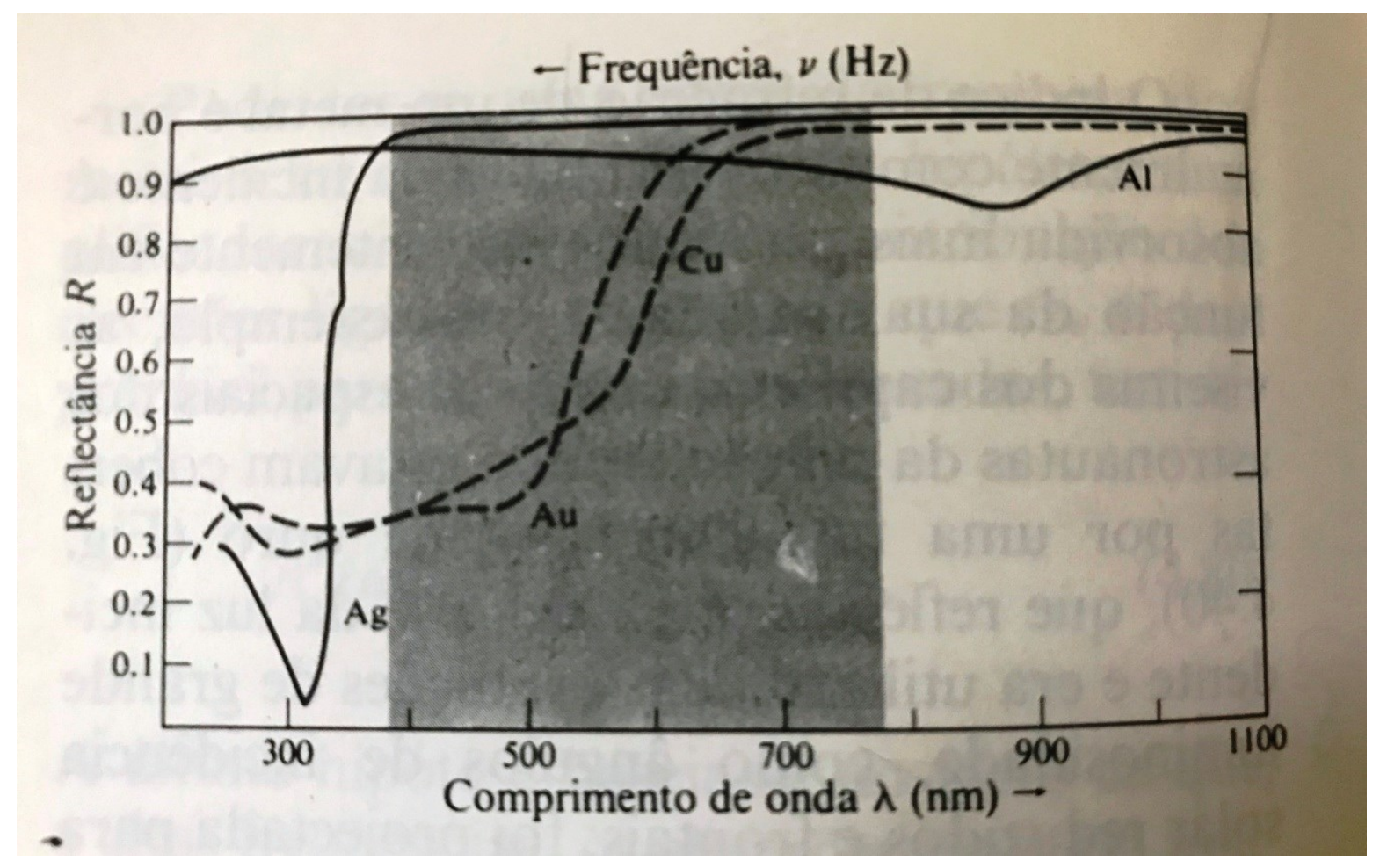

Fig. 9 - Reflectância para a prata, o cobre, o ouro e o alumínio (HECHT, 1987, p.122).

processo de formação de alunos (EM), professores de física (licenciatura) e futuros cientistas (bacharelado), a distinção explícita das facetas fenomenológica e teórica da física, em geral, não é feita. $\mathrm{O}$ uso das imagens trazidas pelos modelos tem papel fundamental na construção da ciência e criam um campo fértil para propiciar a capacidade de abstração, tão essencial para o desenvolvimento do pensamento científico (BACHELARD, 1996). Uma aula tradicional, expositiva e apenas teórica, transmite uma ideia da física de maneira parcial e incompleta. Neste trabalho, mostramos como essas dimensões do conhecimento podem ser abordadas num caso concreto e podem renovar nossa relação com a física.

Com o desenvolvimento da física, o grau de complexidade dos modelos aumentou e o que Bachelard chama de fenomenotécnica (BACHELARD, 1996, p. 73) se torna mais evidente nas pesquisas atuais, onde as dimensões teórica e fenomenológica do conhecimento científico se distinguem mais nitidamente. Desenvolvemos o modelo de Drude em detalhes para enfatizar como ele se utiliza de aspectos teóricos para explicar fenômenos eletromagnéticos, advindos da interação da luz com a matéria. A representação dessa ideia, apresentada de maneira genérica na Fig. 1, pode ser especificada para o modelo de Drude (ver Fig. 11), que tem o importante papel de mediador entre a teoria eletromagnética e os fenômenos tão conhecidos e presentes do nosso cotidiano. 


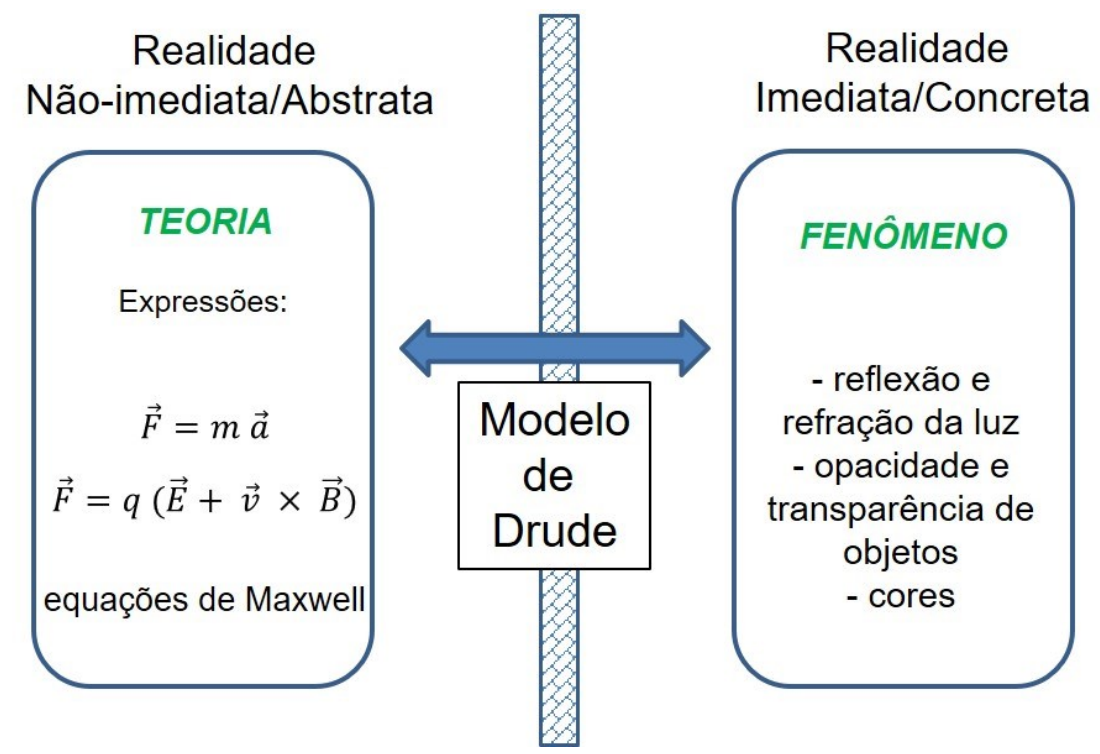

Fig. 10-Modelo de Drude e as dimensões teórica e fenomenológica.

Procuramos aplicar os resultados obtidos com o modelo de Drude na explicação de alguns fenômenos. Mesmo de posse da teoria, suas expressões e significados dos conceitos, não é uma tarefa fácil entender o mundo à nossa volta. Esta dificuldade é intrínseca à natureza do conhecimento, já que o mundo tem as dimensões diferentes, que procuramos enfatizar neste trabalho. O mundo sensível é feito de fenômenos e para termos suas explicações, é necessário um esforço mental específico, ao qual não estamos acostumados, provavelmente por falta de treino durante nossa formação. Parte deste trabalho teve como objetivo mostrar que a física é muito mais do que várias facetas separadas. Ela carrega uma visão de mundo muito ampla e o entendimento das leis que regem o comportamento de tudo que tange os mundos da matéria e da energia.

Uma reflexão um pouco mais profunda acerca da natureza das dificuldades do ensino pode levar a uma consciência da importância da introdução de elementos epistemológicos na abordagem de conteúdos específicos. Muitas vezes, achamos que o problema poderia estar no sistema educacional, tão somente. No entanto, mostramos o quanto 'o saber física' é inesgotável e que isso é uma característica muito forte do conhecimento científico. Por isso, parte da dificuldade do ensino de física (e do aprendizado) tem origem em aspectos intrínsecos à natureza do conhecimento, ou seja, pertence ao campo da epistemologia e não apenas da didática.

\section{Referências bibliográficas}

BACHELARD, G. A Filosofia do Não. Abril Cultural, 1978.

BACHELARD, G. A formação do espírito científico. $5^{\text {a }}$ reimpressão. Contraponto, 1996. 
BECHARA, M. J.; DUARTE, J. L. M.; ROBILOTTA, M. R.; VASCONCELOS, S. S. Física 4. Apostila do curso de Bacharelado. Universidade de São Paulo, 2018.

BREWE, E.; SAWTELLE, V. Modelling instruction for university physics: examining the theory in practice. European Journal of Physics, v. 39, 054001, 2018.

BUNGE, M. Teoria e Realidade. Perspectiva, 1974.

CASSIRER, E. Symbol, Myth and Culture: Essays and lectures of Ernst Cassirer. New Haven London: Yale University Press, 1979.

GRECA, I. M.; MOREIRA, M. A. Mental, physical, and mathematical models in the teaching and learning of physics. Science Education, v. 86, p. 106-121, 2001.

HANSSON, L.; LEDEN, L. Working with the nature of science in physics class: turning 'ordinary' classroom situations into nature of science learning situations. Physics Education, v. 51, 055001, 2016.

HECHT, E. Óptica. Wesley Publishing Company, INC, 1987.

HESTENES, D. Notes for a Modeling Theory of Science, Cognition and Instruction. GIREP 2006 - INTERNATIONAL CONFERENCE. University of Amsterdam, Netherlands, August 2006.

HÖTTECKE, D; HENKE, A; RIESS, F. Implementing History and Philosophy in Science Teaching: Strategies, Methods, Results and Experiences from the European HIPST Project. Science \& Education, dez, 2010.

KNELLER, G. F. A ciência como atividade humana. Editora Zahar/Edusp, 1980.

KNEUBIL, F. B. Models in physics teaching: an approach to highlight the nature of knowledge. Physics Education, v. 51, 065008, 2016.

KNEUBIL, F. B.; KARAM, R. Keyhole: Equal signs as bridges between the phenomenological and theoretical dimensions. Revista Brasileira de Ensino de Física, v. 39, n. 2, e2302, 2017.

KNEUBIL, F. B. As Facetas do Conhecimento Físico e um Modelo: a Luz e a Matéria. 2006. Dissertação (Mestrado em Ensino de Física) - IFUSP, São Paulo. 
KOPONEN, I. Models and modelling in physics education: a critical re-analysis of philosophical underpinnings and suggestions for revisions. Science \& Education, v. 16, n. 7, p.751-773, August 2007.

KRANJC, T. Understanding basic physical concepts - which? The modeling of Real World Phenomena Based on Laws of Physics. GIREP 2006 - INTERNATIONAL CONFERENCE. University of Amsterdam, Netherlands, August 2006.

MATTHEWS, M. Models in science and in science education: an introduction. Science \& Education, v.16, p. 647-652, 2007.

MATTHEWS, M. Science Teaching: The Role of History and Philosophy of Science. New York: Routledge, 1994.

PÉREZ, C. A. S. O Modelo do Elétron Livre de Drude Completa 100 Anos. Caderno Brasileiro de Ensino de Física, v. 17, n. 3, p. 348-359, 2000.

PIETROCOLA, M. Reflexões Histórico-Epistemológicas e o Ensino de Ciências. Texto apresentado como requisito para concurso de Livre Docência. Faculdade de Educação da USP, 2004.

RUDGE, D.; HOWE, E. An explicit and refletive approach to the use of history to promote understanding of the nature of science. Science \& Education, v.18, p. 561-580, 2009.

SCARINCI, A., L.; MARINELI, F. O Modelo Ondulatório da Luz para Explicar as causas da Cor. Revista Brasileira de Ensino de Física, v. 36, n. 1, p. 1309, 2014.

TSEITLIN, M.; GALILI, I. Models in physics teaching: arguing a broader view. GIREP 2006 - INTERNATIONAL CONFERENCE. University of Amsterdam, Netherlands, August 2006.

VILLANI, A; DIAS, V.; VALADARES, J. The Development of Science Education Research in Brazil and Contributions from the History and Philosophy of Science. International Journal of Science Education, v. 32, p. 907-937, 2009. 


\section{Apêndice 1}

Apresentamos os valores das funções $\sigma, \varepsilon, k, \beta, \eta$ e $\delta=1 / \beta$ para um conjunto grande de frequências. Na tabela mostramos os valores de todas elas para o caso do cobre. Os gráficos dessas funções estão mostrados em seguida à tabela (KNEUBIL, 2006, p. 85).

\begin{tabular}{|c|c|c|c|c|c|c|}
\hline$\omega(\mathrm{Hz})$ & $\sigma\left(\Omega^{-1} m^{-1}\right)$ & $\epsilon(F / m)$ & $\mathrm{k}\left(m^{-1}\right)$ & $\beta\left(m^{-1}\right)$ & $\eta(r a d)$ & $\delta(m)$ \\
\hline 1 & $5,880 \mathrm{E}+07$ & $-1,23099 \mathrm{E}-06$ & $6,077 \mathrm{E}+00$ & $6,077 \mathrm{E}+00$ & $7,854 \mathrm{E}-01$ & $1,646 \mathrm{E}-01$ \\
\hline $1 \mathrm{E}+1$ & $5,880 \mathrm{E}+07$ & $-1,23099 \mathrm{E}-06$ & $1,922 \mathrm{E}+01$ & $1,922 \mathrm{E}+01$ & $7,854 \mathrm{E}-01$ & $5,204 \mathrm{E}-02$ \\
\hline $1 \mathrm{E}+2$ & $5,880 \mathrm{E}+07$ & $-1,23099 \mathrm{E}-06$ & $6,077 \mathrm{E}+01$ & $6,077 \mathrm{E}+01$ & $7,854 \mathrm{E}-01$ & $1,646 \mathrm{E}-02$ \\
\hline $1 \mathrm{E}+3$ & $5,880 \mathrm{E}+07$ & $-1,23099 \mathrm{E}-06$ & $1,922 \mathrm{E}+02$ & $1,922 \mathrm{E}+02$ & $7,854 \mathrm{E}-01$ & $5,204 \mathrm{E}-03$ \\
\hline $1 \mathrm{E}+4$ & $5,880 \mathrm{E}+07$ & $-1,23099 \mathrm{E}-06$ & $6,077 \mathrm{E}+02$ & $6,077 \mathrm{E}+02$ & $7,854 \mathrm{E}-01$ & $1,646 \mathrm{E}-03$ \\
\hline $1 \mathrm{E}+5$ & $5,880 \mathrm{E}+07$ & $-1,23099 \mathrm{E}-06$ & $1,922 \mathrm{E}+03$ & $1,922 \mathrm{E}+03$ & $7,854 \mathrm{E}-01$ & $5,204 \mathrm{E}-04$ \\
\hline $1 \mathrm{E}+6$ & $5,880 \mathrm{E}+07$ & $-1,23099 \mathrm{E}-06$ & $6,077 \mathrm{E}+03$ & $6,077 \mathrm{E}+03$ & $7,854 \mathrm{E}-01$ & $1,646 \mathrm{E}-04$ \\
\hline $1 \mathrm{E}+7$ & $5,880 \mathrm{E}+07$ & $-1,23099 \mathrm{E}-06$ & $1,922 \mathrm{E}+04$ & $1,922 \mathrm{E}+04$ & $7,854 \mathrm{E}-01$ & $5,204 \mathrm{E}-05$ \\
\hline $1 \mathrm{E}+8$ & $5,880 \mathrm{E}+07$ & $-1,23099 \mathrm{E}-06$ & $6,077 \mathrm{E}+04$ & $6,077 \mathrm{E}+04$ & $7,854 \mathrm{E}-01$ & $1,646 \mathrm{E}-05$ \\
\hline $1 \mathrm{E}+9$ & $5,880 \mathrm{E}+07$ & $-1,23099 \mathrm{E}-06$ & $1,922 \mathrm{E}+05$ & $1,922 \mathrm{E}+05$ & $7,854 \mathrm{E}-01$ & $5,204 \mathrm{E}-06$ \\
\hline $1 \mathrm{E}+10$ & $5,880 \mathrm{E}+07$ & $-1,23099 \mathrm{E}-06$ & $6,076 \mathrm{E}+05$ & $6,077 \mathrm{E}+05$ & $7,855 \mathrm{E}-01$ & $1,645 \mathrm{E}-06$ \\
\hline $1 \mathrm{E}+11$ & $5,880 \mathrm{E}+07$ & $-1,23099 \mathrm{E}-06$ & $1,920 \mathrm{E}+06$ & $1,924 \mathrm{E}+06$ & $7,864 \mathrm{E}-01$ & $5,198 \mathrm{E}-07$ \\
\hline $1 \mathrm{E}+12$ & $5,877 \mathrm{E}+07$ & $-1,23045 \mathrm{E}-06$ & $6,012 \mathrm{E}+06$ & $6,139 \mathrm{E}+06$ & $7,959 \mathrm{E}-01$ & $1,629 \mathrm{E}-07$ \\
\hline $1 \mathrm{E}+13$ & $5,633 \mathrm{E}+07$ & $-1,17931 \mathrm{E}-06$ & $1,695 \mathrm{E}+07$ & $2,087 \mathrm{E}+07$ & $8,886 \mathrm{E}-01$ & $4,792 \mathrm{E}-08$ \\
\hline $1 \mathrm{E}+14$ & $1,093 \mathrm{E}+07$ & $-2,28717 \mathrm{E}-07$ & $1,247 \mathrm{E}+07$ & $5,503 \mathrm{E}+07$ & $1,348 \mathrm{E}+00$ & $1,817 \mathrm{E}-08$ \\
\hline $1 \mathrm{E}+15$ & $1,339 \mathrm{E}+05$ & $-2,79397 \mathrm{E}-09$ & $1,419 \mathrm{E}+06$ & $5,926 \mathrm{E}+07$ & $1,547 \mathrm{E}+00$ & $1,688 \mathrm{E}-08$ \\
\hline $1 \mathrm{E}+16$ & $1,342 \mathrm{E}+03$ & $-1,92416 \mathrm{E}-11$ & $1,714 \mathrm{E}+05$ & $4,916 \mathrm{E}+07$ & $1,567 \mathrm{E}+00$ & $2,034 \mathrm{E}-08$ \\
\hline $1,50 \mathrm{E}+16$ & $5,964 \mathrm{E}+02$ & $-3,63529 \mathrm{E}-12$ & $1,753 \mathrm{E}+05$ & $3,205 \mathrm{E}+07$ & $1,565 \mathrm{E}+00$ & $3,120 \mathrm{E}-08$ \\
\hline $1,60 \mathrm{E}+16$ & $5,242 \mathrm{E}+02$ & $-2,12342 \mathrm{E}-12$ & $2,016 \mathrm{E}+05$ & $2,613 \mathrm{E}+07$ & $1,563 \mathrm{E}+00$ & $3,827 \mathrm{E}-08$ \\
\hline $1,70 \mathrm{E}+16$ & $4,643 E+02$ & $-8,70406 \mathrm{E}-13$ & $2,788 \mathrm{E}+05$ & $1,778 \mathrm{E}+07$ & $1,555 \mathrm{E}+00$ & $5,625 \mathrm{E}-08$ \\
\hline $1,75 \mathrm{E}+16$ & $4,382 E+02$ & $-3,22893 \mathrm{E}-13$ & $4,318 \mathrm{E}+05$ & $1,115 \mathrm{E}+07$ & $1,532 \mathrm{E}+00$ & $8,966 \mathrm{E}-08$ \\
\hline $1,78 \mathrm{E}+16$ & $4,235 \mathrm{E}+02$ & $-1,63026 \mathrm{E}-14$ & $1,580 \mathrm{E}+06$ & $2,997 \mathrm{E}+06$ & $1,086 \mathrm{E}+00$ & $3,337 \mathrm{E}-07$ \\
\hline $1,78 \mathrm{E}+16$ & $4,227 \mathrm{E}+02$ & $-3,84713 \mathrm{E}-16$ & $2,157 \mathrm{E}+06$ & $2,193 \mathrm{E}+06$ & $7,935 \mathrm{E}-01$ & $4,561 \mathrm{E}-07$ \\
\hline $1,78 \mathrm{E}+16$ & $4,227 \mathrm{E}+02$ & 0 & $2,175 \mathrm{E}+06$ & $2,175 \mathrm{E}+06$ & $7,854 \mathrm{E}-01$ & $4,598 \mathrm{E}-07$ \\
\hline $1,79 \mathrm{E}+16$ & $4,188 \mathrm{E}+02$ & $8,24849 \mathrm{E}-14$ & $5,818 \mathrm{E}+06$ & $8,092 \mathrm{E}+05$ & $1,382 \mathrm{E}-01$ & $1,236 \mathrm{E}-06$ \\
\hline $1 \mathrm{E}+17$ & $1,342 \mathrm{E}+01$ & $8,56908 \mathrm{E}-12$ & $3,281 \mathrm{E}+08$ & $2,569 \mathrm{E}+03$ & $7,830 \mathrm{E}-06$ & $3,893 \mathrm{E}-04$ \\
\hline $1 \mathrm{E}+18$ & $1,342 \mathrm{E}-01$ & $8,84719 \mathrm{E}-12$ & $3,333 \mathrm{E}+09$ & $2,528 \mathrm{E}+01$ & $7,583 \mathrm{E}-09$ & $3,956 \mathrm{E}-02$ \\
\hline $1 \mathrm{E}+19$ & $1,342 \mathrm{E}-03$ & $8,84997 \mathrm{E}-12$ & $3,334 \mathrm{E}+10$ & $2,528 \mathrm{E}-01$ & $7,581 \mathrm{E}-12$ & $3,956 \mathrm{E}+00$ \\
\hline $1 \mathrm{E}+20$ & $1,342 \mathrm{E}-05$ & $8,85 \mathrm{E}-12$ & $3,334 \mathrm{E}+11$ & $2,528 \mathrm{E}-03$ & $7,581 \mathrm{E}-15$ & $3,956 \mathrm{E}+02$ \\
\hline $1 \mathrm{E}+21$ & $1,342 \mathrm{E}-07$ & $8,85 \mathrm{E}-12$ & $3,334 \mathrm{E}+12$ & $2,528 \mathrm{E}-05$ & $7,581 \mathrm{E}-18$ & $3,956 \mathrm{E}+04$ \\
\hline $1 \mathrm{E}+22$ & $1,342 \mathrm{E}-09$ & $8,85 \mathrm{E}-12$ & $3,334 \mathrm{E}+13$ & $2,528 \mathrm{E}-07$ & $7,581 \mathrm{E}-21$ & $3,956 \mathrm{E}+06$ \\
\hline
\end{tabular}



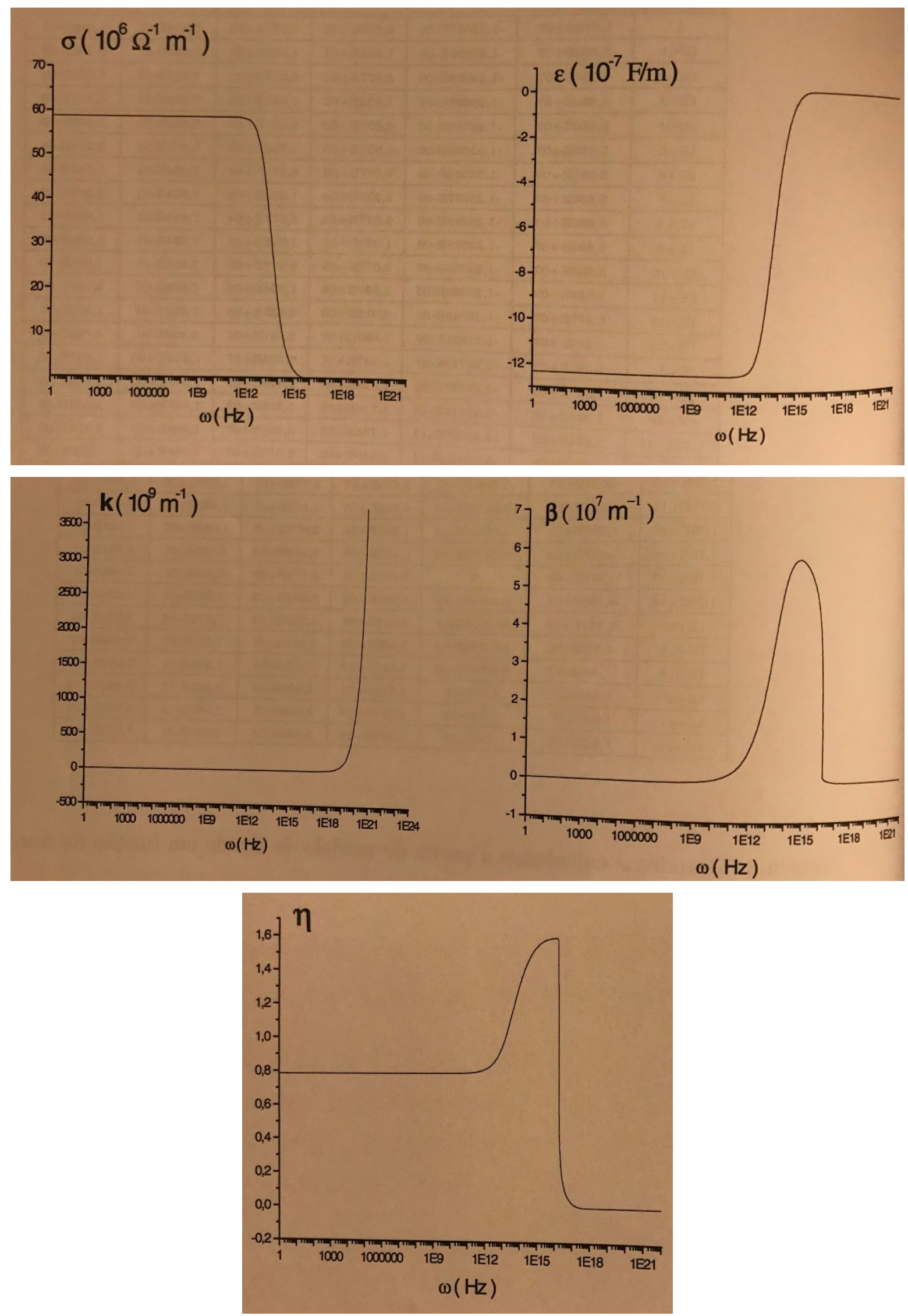

\section{(cc) EY-NC-ND}

Direito autoral e licença de uso: Este artigo está licenciado sob uma Licença Creative Commons. 